\title{
PRIMES IN TUPLES IV: DENSITY OF SMALL GAPS BETWEEN CONSECUTIVE PRIMES
}

\author{
D. A. GOLDSTON, J. PINTZ AND C. Y. YILDIRIM
}

\section{INTRODUCTION}

Let, as usual, $\pi(x)$ denote the number of primes $\leq x$, and $p_{n}$ the $n$-th prime. The prime number theorem says $\pi(x) \sim \frac{x}{\log x}$ as $x \rightarrow \infty$, so that on average $p_{n+1}-p_{n}$ is $\log p_{n}$. In [3] we proved that

$$
\liminf _{n \rightarrow \infty} \frac{p_{n+1}-p_{n}}{\log p_{n}}=0
$$

The main result of this paper is

Theorem 1 For any fixed $\eta>0$, we have

$$
\#\left\{p_{n} \leq x ; p_{n+1}-p_{n} \leq \eta \log p_{n}\right\} \gg_{\eta} \pi(x),
$$

i.e. the small gaps between primes attested by the proof of (1.1) in fact constitute a positive proportion of the set of all gaps between consecutive primes.

The course of the proof leads to Theorem 2 involving the explicit estimate (3.26) below. In the last section we conditionally find stronger versions of (3.26), based upon assuming more than what the Bombieri-Vinogradov theorem provides. These results also provide within the framework of our method a new quantitative manifestation of the effect of the extent of assumed information on how well the primes are distributed in arithmetic progressions. Theorem 3 in the fourth section expresses that very small gaps are sparse. A rather qualitative concise version of the results of this paper has been presented in [5].

Concerning our subject matter it is conjectured (see [14, 15]) that, given $0 \leq$ $\alpha<\beta$, as $x \rightarrow \infty$ we have

$$
\#\left\{p_{n} \leq x ; p_{n+1} \in\left(p_{n}+\alpha \log p_{n}, p_{n}+\beta \log p_{n}\right)\right\} \sim \pi(x) \int_{\alpha}^{\beta} e^{-t} d t .
$$

Gallagher's 2] calculation shows that this conjecture can be deduced from the Hardy-Littlewood prime $k$-tuples conjecture.

A bibliography of former results for the limit in (1.1) was given in [3]. Before [3] the best known result for this limit was

$$
\liminf _{n \rightarrow \infty} \frac{p_{n+1}-p_{n}}{\log p_{n}} \leq 0.2484 \ldots,
$$

Date: March 31, 2011.

The first author was supported by NSF; this study was done while the second and third authors were members at the Institute for Advanced Studies, Princeton during Fall 2009 and were supported by the Oswald Veblen Fund; the second author also acknowledges the partial support of ERC-AdG. No.228005. 
due to Maier [11. In his proof Maier employed specially constructed thin sets in which the density of primes is larger by a factor of $e^{\gamma}$ than on average, and paralleling the Bombieri-Davenport-Huxley method (1], [10]) with necessary modifications, he attained (1.4) which is $e^{-\gamma}$ times the result of this former method. Since Maier's method involved working within a thin set of integers, the small gaps indicated by (1.4) could not provide a positive proportion of all gaps. The second best result was $\leq \frac{1}{4}$ by Goldston and Ylldırım [7. The origin of its method also being the Bombieri-Davenport proof, the small gaps found in [7] were shown to occur in a positive proportion of all cases.

Let us recall briefly how the result (1.1) was obtained. Consider the $k$-tuple

$$
\mathcal{H}=\left\{h_{1}, h_{2}, \ldots, h_{k}\right\} \text { with distinct integers } h_{1}, \ldots, h_{k} \in[1, h],
$$

and for a prime $p$ denote by $\nu_{p}(\mathcal{H})$ the number of distinct residue classes modulo $p$ occupied by the entries of $\mathcal{H}$. The singular series associated with $\mathcal{H}$ is defined as

$$
\mathfrak{S}(\mathcal{H}):=\prod_{p}\left(1-\frac{1}{p}\right)^{-k}\left(1-\frac{\nu_{p}(\mathcal{H})}{p}\right),
$$

the product being convergent because $\nu_{p}(\mathcal{H})=k$ for $p>h$. We say that $\mathcal{H}$ is admissible if

$$
P_{\mathcal{H}}(n):=\left(n+h_{1}\right)\left(n+h_{2}\right) \cdots\left(n+h_{k}\right)
$$

is not divisible by a fixed prime number for every $n$, which is equivalent to $\nu_{p}(\mathcal{H}) \neq p$ for all $p$ and therefore also to $\mathfrak{S}(\mathcal{H}) \neq 0$. That $\left\{n+h_{1}, n+h_{2}, \ldots, n+h_{k}\right\}$ is a prime tuple, i.e. each entry is prime, is equivalent to $P_{\mathcal{H}}(n)$ being a product of $k$ primes. Since the generalized von Mangoldt function

$$
\Lambda_{k}(m):=\sum_{d \mid m} \mu(d)\left(\log \frac{m}{d}\right)^{k}
$$

vanishes when $m$ has more than $k$ distinct prime factors, the quantity

$$
\frac{1}{k !} \sum_{\substack{d \mid P_{\mathcal{H}}(n) \\ d \leq R}} \mu(d)\left(\log \frac{R}{d}\right)^{k}
$$

with the truncation $d \leq R$ may be employed in a roughly correct detection of prime tuples (the contribution from proper prime power factors is negligible; $1 / k$ ! is a normalization factor). One crucial idea is to give up trying to count tuples consisting of primes exclusively, but rather also include tuples with primes in many entries. This brings about the use of

$$
\Lambda_{R}(n ; \mathcal{H}, \ell):=\frac{1}{(k+\ell) !} \sum_{\substack{d \mid \mathcal{P}_{\mathcal{H}}(n) \\ d \leq R}} \mu(d)\left(\log \frac{R}{d}\right)^{k+\ell}, \quad(0 \leq \ell<k)
$$

so as to allow counting those $P_{\mathcal{H}}(n)$ which have at most $k+\ell$ distinct prime factors.

Let

$$
\theta(n):= \begin{cases}\log n & \text { if } n \text { is prime } \\ 0 & \text { otherwise }\end{cases}
$$

and

$$
\Theta(n, h):=\sum_{1 \leq h_{0} \leq h} \theta\left(n+h_{0}\right)
$$


The proof of (1.1) is achieved by showing the positivity of the quantity

$$
S_{R}(N, k, \ell, h):=\sum_{N<n \leq 2 N}(\Theta(n, h)-\log 3 N)\left(\sum_{\substack{\mathcal{H} \subset[1, h] \\|\mathcal{H}|=k}} \Lambda_{R}(n ; \mathcal{H}, \ell)\right)^{2} .
$$

Here, as $N \rightarrow \infty$, for a result of the type (1.1) we need $\epsilon \log N \ll h \ll \log N$ with an arbitrarily small but fixed $\epsilon>0$, and the larger the truncation level $R$ is relative to $N$ the better detection will be provided by (1.8). The tuple size $k$ is taken to be arbitrarily large but fixed. In fact for the proof of (1.1) it suffices to consider the simpler expression where the inner sum consists only of the diagonal terms $\Lambda_{R}^{2}(n ; \mathcal{H}, \ell)$, and a modified version of this will be used in Section 3. The expression in (1.11) is needed for achieving a better result in the case of the gaps $p_{n+r}-p_{n}$ with $r \geq 2$ in [3] and for a stronger quantitative version of (1.1) in [4].

The information on primes, beyond the prime number theorem, that is of key importance in our studies is the level of distribution of primes in arithmetic progressions. We say that the primes satisfy a level of distribution $\vartheta$ if

$$
\sum_{q \leq Q} \max _{\substack{a \\(a, q)=1}}\left|\sum_{\substack{p: \text { prime } \\ p \leq N \\ p \equiv a(\bmod q)}} \log p-\frac{N}{\phi(q)}\right| \ll_{\epsilon, A} \frac{N}{(\log N)^{A}}
$$

holds for any $A>0$ and any $\epsilon>0$ with

$$
Q=N^{\vartheta-\epsilon} .
$$

According to the Bombieri-Vinogradov theorem, for any $A>0$ there is a $B=B(A)$ such that (1.12) holds with $Q=N^{\frac{1}{2}}(\log N)^{-B}$, so that the primes are known to have level of distribution $\frac{1}{2}$. The Elliott-Halberstam conjecture is that the primes have level of distribution 1 .

The following are special cases of some results from [3] which are relevant to our purpose in this article. For an admissible $k$-tuple $\mathcal{H}$, we have

$$
\sum_{n \leq N} \Lambda_{R}(n ; \mathcal{H}, \ell)^{2} \sim\left(\begin{array}{c}
2 \ell \\
\ell
\end{array}\right) \frac{(\log R)^{k+2 \ell}}{(k+2 \ell) !} \mathfrak{S}(\mathcal{H}) N,
$$

as $R, N \rightarrow \infty$, for $R \ll N^{\frac{1}{2}}(\log N)^{-8 M}$ where $M=k+\ell$, and $h \leq R^{C}$ for any given constant $C>0$. In the situation of weighting with the primes, for $1 \leq h_{0} \leq h$ writing $m=1$ when $h_{0} \in \mathcal{H}$ and $m=0$ when $h_{0} \notin \mathcal{H}$, if $\mathcal{H} \cup\left\{h_{0}\right\}$ is admissible we have

$$
\sum_{N \leq n} \theta\left(n+h_{0}\right) \Lambda_{R}(n ; \mathcal{H}, \ell)^{2} \sim\left(\begin{array}{c}
2(\ell+m) \\
\ell+m
\end{array}\right) \frac{\mathfrak{S}\left(\mathcal{H} \cup\left\{h_{0}\right\}\right)}{(k+2 \ell+m) !} N(\log R)^{k+2 \ell+m}
$$

as $R, N \rightarrow \infty$, provided that $R \ll_{M} N^{\frac{1}{4}}(\log N)^{-B(M)}$ for a sufficiently large positive constant $B(M)$, and $h \leq R$. The upper bound for $R$ is forced by the dependence of the proof of (1.15) on the Bombieri-Vinogradov theorem, and for the unconditional results in [3], taking $R=N^{\frac{1}{4}-\epsilon}$ suffices. More generally, (1.15) holds with $R \ll N^{\frac{\vartheta}{2}-\epsilon}$ and $h \leq R^{\epsilon}$ for any $\epsilon>0$, assuming that the primes have level of distribution $\vartheta$ with a fixed $\vartheta \in\left[\frac{1}{2}, 1\right]$.

The proof of (1.14) and (1.15) may be outlined as follows. Upon writing the left-hand sides explicitly by substituting (1.8), the sum over $n$ is carried to the innermost position and easily evaluated. Then a Mellin transform converts the 
expressions into integrals over vertical lines in the complex plane. The integrands contain Dirichlet series which encode the arithmetic information from the tuples. The integrals are evaluated by shifting the lines of integration appropriately and by calculating the residues and the bounds for the integrals over the new contours.

For the calculation of $S_{R}(N, k, \ell, h)$, the general versions of (1.14) and (1.15) are employed in the expression on the right-hand side of (1.11), and then Gallagher's 2 result

$$
\sum_{\substack{\mathcal{H} \subset[1, h] \\|\mathcal{H}|=k}} \mathfrak{S}(\mathcal{H}) \sim h^{k} \quad \text { for fixed } k \text { as } h \rightarrow \infty
$$

(where each set is counted $k$ ! times due to all of its permutations) is needed to complete the calculation. The parameters which appear in this process are chosen judiciously, in particular $k$ has to be arbitrarily large but fixed and the optimal order of magnitude of the integer $\ell$ turns out to be $\sqrt{k}$.

The proof of the positive proportion result in 7] uses an argument which depends on the calculation of the fourth moment of prime tuple approximants. If that argument is adapted straightforwardly to the approach which led to (1.1), for a proof of Theorem 1 one needs to show that

$$
\sum_{N<n \leq 2 N}\left(\sum_{\substack{\mathcal{H} \subset[1, h] \\|\mathcal{H}|=k}} \Lambda_{R}(n ; \mathcal{H}, \ell)\right)^{4} \ll N(\log N)^{4 k+4 \ell}
$$

However, upon some calculations, the truth of this seems to be questionable.

\section{Some PRELIMINARIES}

The lack of success from a direct use of results from [3] for the proof of a positive proportion result notwithstanding, a version of (1.14) and (1.15) in which the $n$ with $P_{\mathcal{H}}(n)$ having small prime factors are discounted vouchsafes the solution. We define

$$
\mathcal{P}(x):=\prod_{p_{n} \leq x} p_{n}
$$

We shall use the following results which are consequences of (1.14), (1.15) and Lemmas 4 and 5 of Pintz's work [12.

Proposition 1 For $N^{c_{1}} \leq R \leq N^{\frac{1}{2+\delta}}(\log N)^{-c_{2}}$ where $c_{1}$ and $c_{2}$ are suitably chosen constants depending on $k$ and $\ell \asymp \sqrt{k}\left(c_{1}\right.$ can be taken to be $\frac{1}{5}$ and $c_{2}$ is sufficiently large), $\delta>0$ small compared to $k^{-\frac{3}{2}}, \mathcal{H}$ admissible with $h \ll \log R$ and $h \rightarrow \infty$ with $N$, we have

$$
\sum_{\substack{N<n \leq 2 N \\
\left(P \mathcal{H}(n), \mathcal{P}\left(R^{\delta}\right)\right)=1}} \Lambda_{R}(n ; \mathcal{H}, \ell)^{2} \sim\left(1+O\left(k^{3} \delta^{2}\right)\right)\left(\begin{array}{c}
2 \ell \\
\ell
\end{array}\right) \frac{\mathfrak{S}(\mathcal{H})}{(k+2 \ell) !} N(\log R)^{k+2 \ell} .
$$

Proposition 2 Upon the conditions of Proposition 1 and the notation introduced in connection with (1.15), if the level of distribution of primes is $\vartheta \geq \frac{1}{2}$, then for $N^{c_{1}} \leq R \leq N^{\frac{\vartheta-\epsilon}{(2+\delta)}}(\log N)^{-c_{2}}\left(\epsilon>0\right.$ arbitrarily small but fixed) and $\mathcal{H} \cup\left\{h_{0}\right\}$ 
admissible, we have

$$
\begin{aligned}
& \sum_{\substack{N<n \leq 2 N \\
\left(P_{\mathcal{H}}(n), \mathcal{P}\left(R^{\delta}\right)\right)=1}} \theta\left(n+h_{0}\right) \Lambda_{R}(n ; \mathcal{H}, \ell)^{2} \\
& \quad \sim\left(1+O\left(k^{3} \delta^{2}\right)\right)\left(\begin{array}{c}
2(\ell+m) \\
\ell+m
\end{array}\right) \frac{\mathfrak{S}\left(\mathcal{H} \cup\left\{h_{0}\right\}\right)}{(k+2 \ell+m) !} N(\log R)^{k+2 \ell+m} ;
\end{aligned}
$$

in case $\mathcal{H} \cup\left\{h_{0}\right\}$ is not admissible, the right-hand side of (2.3) is o $\left(N(\log R)^{k+2 \ell+m}\right)$.

Proof: $\quad$ From [12], along with (1.14), (1.15) these results are obvious except that the present error term $O\left(k^{3} \delta^{2}\right)$ meant with an absolute constant comes out as $O(\delta)$ with the constant implied depending on $k$ and $\ell$. Since we shall use the dependence on $k$ and $\ell$ of the error term, we give its proof. An examination of the proof of Pintz's Lemma 3 reveals that we need to have more precise versions of $(6.17),(6.18)$, (6.25) and (6.26) of [12. First we evaluate

$$
\begin{aligned}
\mathcal{T}_{q, 1}(1+\alpha) & :=\frac{1}{\ell !}\left[\left(\frac{d}{d \xi}\right)^{\ell}\left(\frac{(1+\alpha+\xi)^{k+2 \ell}}{(1+\xi)^{k}}\right)\right]_{\xi=0} \\
& =(1+\alpha)^{k+\ell} \sum_{m=0}^{\ell}\left(\begin{array}{c}
2 \ell-m \\
\ell
\end{array}\right)\left(\begin{array}{c}
k+m-1 \\
m
\end{array}\right)(-\alpha)^{m} .
\end{aligned}
$$

This indicates that it would be opportune to restrict $|\alpha|$ to values small compared to $\frac{1}{k}$. Assuming this, and recalling that $\ell \asymp \sqrt{k}$, from $(2.4)$ we see that

$$
\mathcal{T}_{q, 1}(1+\alpha)=\left(\begin{array}{c}
2 \ell \\
\ell
\end{array}\right)\left(1+\left(\frac{k}{2}+\ell\right) \alpha+\left(\frac{k^{2}}{8}+\frac{k \ell}{2}-\frac{3 k}{8}+\frac{\ell^{2}}{2}-\frac{\ell}{2}-\frac{k(k+1)}{8(2 \ell-1)}\right) \alpha^{2}+O\left((k \alpha)^{3}\right)\right) .
$$

We will denote the coefficient of $\alpha^{2}$ in the last line as $K$. This is used in (6.8) of 12. We recall that the prime number $q=R^{\beta}$ in the statement of Lemma 3. In the last factor of the integrand of (6.8) there are four terms. With the notation introduced in (6.11) of [12] we have the following. The first term has $R_{1}=R_{2}=R$, so that $\alpha=0$, and we get the contribution

$$
\left(\begin{array}{c}
2 \ell \\
\ell
\end{array}\right) \frac{(\log R)^{k+2 \ell}}{(k+2 \ell) !} G_{q}(0,0) \text {. }
$$

The second term has $R_{1}=R / q, R_{2}=R$, so that $\alpha=-\beta$, and we get the contribution

$$
\left(\begin{array}{c}
2 \ell \\
\ell
\end{array}\right) \frac{(\log R)^{k+2 \ell}}{(k+2 \ell) !} G_{q}(0,0)\left[1-\left(\frac{k}{2}+\ell\right) \beta+K \beta^{2}+O\left((k \beta)^{3}\right)\right] .
$$

The third term has $R_{1}=R, R_{2}=R / q$, so that $\alpha=\frac{\beta}{1-\beta}$, and we get the contribution

$$
\left(\begin{array}{c}
2 \ell \\
\ell
\end{array}\right) \frac{\left(\log R^{1-\beta}\right)^{k+2 \ell}}{(k+2 \ell) !} G_{q}(0,0)\left[1+\left(\frac{k}{2}+\ell\right) \frac{\beta}{1-\beta}+K\left(\frac{\beta}{1-\beta}\right)^{2}+O\left((k \beta)^{3}\right)\right] .
$$

The fourth term has $R_{1}=R_{2}=R / q$, so that $\alpha=0$, and we get the contribution

$$
\left(\begin{array}{c}
2 \ell \\
\ell
\end{array}\right) \frac{\left(\log R^{1-\beta}\right)^{k+2 \ell}}{(k+2 \ell) !} G_{q}(0,0) \text {. }
$$


Combining these as in (6.8) we obtain

$$
\left(\begin{array}{c}
2 \ell \\
\ell
\end{array}\right) \frac{(\log R)^{k+2 \ell}}{(k+2 \ell) !} G_{q}(0,0)\left[\left(\frac{k^{2}}{4}+k \ell+\ell^{2}+\frac{k}{4}+\frac{k(k+1)}{4(2 \ell-1)}\right) \beta^{2}+O\left((k \beta)^{3}\right)\right]
$$

in place of the main term of (6.25) of [12]. Hence in the new version for (6.1) of [12], instead of $\frac{\beta}{q}$ we have

$$
\begin{aligned}
& \frac{\nu_{q}(\mathcal{H})}{q} \frac{G_{q}(0,0)}{G(0,0)}\left[\left(\frac{k^{2}}{4}+k \ell+\ell^{2}+\frac{k}{4}+\frac{k(k+1)}{4(2 \ell-1)}\right) \beta^{2}+O\left((k \beta)^{3}\right)\right] \\
& \quad=\frac{\nu_{q}(\mathcal{H})}{q}\left(1-\frac{\nu_{q}(\mathcal{H})}{q}\right)^{-1}\left(\frac{k^{2} \beta^{2}}{4}+\text { smaller terms }\right),
\end{aligned}
$$

i.e. we can express the new version of Lemma 3 of $[12$ as

$$
\sum_{\substack{N<n \leq 2 N \\ q \mid P_{\mathcal{H}}(n)}} \Lambda_{R}(n ; \mathcal{H}, \ell)^{2} \leq \frac{\nu_{q}(\mathcal{H})}{q-\nu_{q}(\mathcal{H})} \frac{k^{2} \beta^{2}}{3} \sum_{N<n \leq 2 N} \Lambda_{R}(n ; \mathcal{H}, \ell)^{2} .
$$

Here $N^{c_{1}} \leq R \leq N^{\frac{1}{2+\delta}}(\log N)^{-c_{2}}, q$ is a prime number for which we write $q=R^{\beta}$, with $0<\beta \leq \delta$ where $\delta$ is small compared to $k^{-\frac{3}{2}}$ say, $k$ is sufficiently large, and $\ell \asymp \sqrt{k}$.

We know that $\nu_{q}(\mathcal{H}) \leq \min (q-1, k)$ since $\mathcal{H}$ is admissible. For $q \leq k$, we have $\nu_{q}(\mathcal{H}) \leq q-1$, so that $\frac{\nu_{q}(\mathcal{H})}{q-\nu_{q}(\mathcal{H})} \leq q-1$. For $q>k$, we take $\nu_{q}(\mathcal{H}) \leq k$, so that $\frac{\nu_{q}(\mathcal{H})}{q-\nu_{q}(\mathcal{H})} \leq \frac{k}{q-k}$. Summing over all primes $q \leq R^{\delta}$ we obtain a new version of Lemma 4 of [12] as

$$
\sum_{\substack{\left.N<n \leq 2 N \\ P_{\mathcal{H}}(n), \mathcal{P}\left(R^{\delta}\right)\right)>1}} \Lambda_{R}(n ; \mathcal{H}, \ell)^{2} \leq \frac{k^{3} \delta^{2}}{4} \sum_{N<n \leq 2 N} \Lambda_{R}(n ; \mathcal{H}, \ell)^{2}
$$

if $k$ is large enough. We see that we have to choose $\delta$ small enough so that $k^{3} \delta^{2}$ will be small. Now by (1.14) and (1.15) we immediately obtain (2.2). When there is the twisting with primes the proof runs similarly and Proposition 2 also follows.

\section{Proof of Theorem 1; Theorem 2}

In order to prove Theorem 1 we need to show the inequality

$$
\sum_{\substack{N<p_{j} \leq 2 N \\ p_{j+1}-p_{j} \leq h}} 1 \gg \pi(N) \sim \frac{N}{\log N}, \quad(N \rightarrow \infty),
$$

for

$$
h=\eta \log N, \quad \eta>0 \text { arbitrarily small but fixed. }
$$

Let

$$
Q(N, h):=\sum_{\substack{N<n \leq 2 N \\ \pi(n+h)-\pi(n)>1}} 1 .
$$


If $n$ is an integer for which $\pi(n+h)-\pi(n)>1$, then there must be a $j$ such that $n<p_{j}$ and $p_{j+1} \leq n+h$. Thus $p_{j+1}-p_{j}<h$ and $p_{j+1}-h \leq n<p_{j}$, so that there are less than $\lfloor h\rfloor$ such integers $n$ corresponding to each such gap. Therefore

$$
Q(N, h) \leq h \sum_{\substack{N<p_{j} \leq 2 N \\ p_{j+1}-p_{j} \leq h}} 1+O\left(N e^{-c \sqrt{\log N}}\right),
$$

where we have used the prime number theorem with error term to remove the prime gaps which overlap the endpoints. (This is explicitly shown in [6]).

Instead of $S_{R}$ which was defined in (1.11), we will work with

$$
\tilde{S}_{R}:=\frac{1}{N(h \log R)^{k}} \sum_{N<n \leq 2 N}(\Theta(n, h)-\log 3 N)\left(\sum_{\mathcal{H}}^{*} \Lambda_{R}^{2}(n ; \mathcal{H}, \ell)\right),
$$

where

$$
\sum_{\mathcal{H}}^{*}:=\sum_{\begin{array}{c}
\mathcal{H} \subset[1, h],|\mathcal{H}|=k \\
\mathcal{H}: \text { admisible } \\
\left(P_{\mathcal{H}}(n), \mathcal{P}\left(R^{\delta}\right)\right)=1
\end{array}} .
$$

We note that, as a function of $\eta, k$ and $\ell$ will be chosen sufficiently large but fixed, and $\delta>0$ will be chosen sufficiently small but fixed (see (3.22) below).

From (3.5) we have, when $N$ is sufficiently large,

$$
\begin{aligned}
\tilde{S}_{R} & \leq \frac{1}{N(h \log R)^{k}} \sum_{\substack{N<n \leq 2 N \\
\Theta(n, h) \geq \frac{3}{2} \log N}} \Theta(n, h) \sum_{\mathcal{H}}^{*} \Lambda_{R}^{2}(n ; \mathcal{H}, \ell) \\
& \leq \frac{1}{N(h \log R)^{k}}\left\{\sum_{\substack{N<n \leq 2 N \\
\Theta(n, h) \geq \frac{3}{2} \log N}} 1\right\}^{\frac{1}{2}}\left\{\sum_{N<n \leq 2 N}(\Theta(n, h))^{2}\left(\sum_{\mathcal{H}}^{*} \Lambda_{R}^{2}(n ; \mathcal{H}, \ell)\right)^{2}\right\}^{\frac{1}{2}} \\
& =\frac{Q(N, h)^{\frac{1}{2}}}{N(h \log R)^{k}} I^{\frac{1}{2}}
\end{aligned}
$$

where

$$
\begin{array}{ll}
I=\sum_{\substack{1 \leq h^{\prime}, h^{\prime \prime} \leq h \\
\mathcal{H}_{i} \subset[1, h],\left|\mathcal{H}_{i}\right|=k \\
\mathcal{H}_{i}: \text { admissible } \\
i=1,2}} \sum_{\substack{N<n \leq 2 N \\
\left(P_{\mathcal{H}_{1} \cup \mathcal{H}_{2}}(n), \mathcal{P}\left(R^{\delta}\right)\right)=1}} \theta\left(n+h^{\prime}\right) \theta\left(n+h^{\prime \prime}\right) \\
& \times \Lambda_{R}^{2}\left(n ; \mathcal{H}_{1}, \ell\right) \Lambda_{R}^{2}\left(n ; \mathcal{H}_{2}, \ell\right) .
\end{array}
$$

Here for a number $n$ to make a nonzero contribution both of $n+h^{\prime}$ and $n+h^{\prime \prime}$ must be prime, so that $\left(\left(n+h^{\prime}\right)\left(n+h^{\prime \prime}\right), \mathcal{P}\left(R^{\delta}\right)\right)=1$ and writing $\mathcal{H}_{0}=\left\{h^{\prime}\right\} \cup\left\{h^{\prime \prime}\right\} \cup \mathcal{H}_{1} \cup \mathcal{H}_{2}$ we can re-express the condition on $n$ as $\left(P_{\mathcal{H}_{0}}(n), \mathcal{P}\left(R^{\delta}\right)\right)=1$. We also observe that, since all prime factors of $P_{\mathcal{H}}(n)$ in $\sum_{\mathcal{H}}^{*}$ are greater than $R^{\delta}$, the number of squarefree divisors of $P_{\mathcal{H}}(n)$ is at most $2^{\frac{k \log 3 N}{\delta \log R}}$. So, for any term in $\sum_{\mathcal{H}}^{*}$ we have

$$
\Lambda_{R}(n ; \mathcal{H}, \ell) \leq \frac{2^{\frac{k \log 3 N}{\delta \log R}}}{(k+\ell) !}(\log R)^{k+\ell}
$$


and therefore

$$
I \leq \frac{2^{\frac{4 k \log 3 N}{\delta \log R}}(\log R)^{4(k+\ell)}(\log 3 N)^{2}}{(k+\ell) !^{4}} \sum_{1 \leq h^{\prime}, h^{\prime \prime} \leq h} \sum_{\substack{\mathcal{H}_{i} \subset[1, h],\left|\mathcal{H}_{i}\right|=k \\ \mathcal{H}_{i}: \text { admissible } \\ i=1,2}} \sum_{\substack{N<n \leq 2 N \\\left(P_{\mathcal{H}_{0}}(n), \mathcal{P}\left(R^{\delta}\right)\right)=1}} 1 .
$$

For a given $\mathcal{H}_{0} \subset\{1, \ldots, h\}$ with $\left|\mathcal{H}_{0}\right|=k+r, 0 \leq r \leq k+2$, denoting by $D(k, r)$ the number of quadruples $h^{\prime}, h^{\prime \prime}, \mathcal{H}_{1}, \mathcal{H}_{2}$ corresponding to $\mathcal{H}_{0}$, we re-express (3.10) as

$$
I \leq \frac{2^{\frac{4 k \log 3 N}{\delta \log R}}(\log R)^{4(k+\ell)}(\log 3 N)^{2}}{(k+\ell) !^{4}} \sum_{r=0}^{k+2} D(k, r) \sum_{\left|\mathcal{H}_{0}\right|=k+r} \sum_{\substack{N<n \leq 2 N \\\left(P \mathcal{H}_{0}(n), \mathcal{P}\left(R^{\delta}\right)\right)=1}} 1
$$

We now invoke the main theorem of Selberg's upper bound sieve (Theorem 5.1 of [9] or Theorem 2 in $\S 2.2 .2$ of [8]) that for any set $\mathcal{H}$ and $\delta<\frac{1}{2}$

$$
\sum_{\substack{N<n \leq 2 N \\\left(P_{\mathcal{H}}(n), \mathcal{P}\left(R^{\delta}\right)\right)=1}} 1 \leq \frac{N|\mathcal{H}| ! \mathfrak{S}(\mathcal{H})}{\left(\log R^{\delta}\right)^{|\mathcal{H}|}}(1+o(1)), \quad(N \rightarrow \infty),
$$

which gives upon using (1.16) that

$$
\begin{aligned}
I & \lesssim N \frac{2^{\frac{4 k \log 3 N}{\delta \log R}}(\log R)^{4(k+\ell)}(\log N)^{2}}{(k+\ell) !^{4}} \sum_{r=0}^{k+2} \frac{(k+r) ! D(k, r)}{(\delta \log R)^{k+r}} \sum_{\mathcal{H}_{0} ;\left|\mathcal{H}_{0}\right|=k+r} \mathfrak{S}\left(\mathcal{H}_{0}\right) \\
& \lesssim N \frac{2^{\frac{4 k \log 3 N}{\delta \log R}}(\log R)^{4(k+\ell)}(\log N)^{2}}{(k+\ell) !^{4}} \sum_{r=0}^{k+2}(k+r) ! D(k, r)\left(\frac{h}{\delta \log R}\right)^{k+r}
\end{aligned}
$$

To deal with the inner sum here, first note that

$$
D(k, r):=\frac{k !^{2}(k+r) !\left(k^{4}+3 k^{3}+(3 r+2) k^{2}+4 r k+r^{2}\right)}{r !^{2}(k+2-r) !}
$$

(here the factor $k !^{2}$ comes from the ordering of the elements within the $k$-tuples $\mathcal{H}_{1}$ and $\mathcal{H}_{2}$ ). We skip the proof of (3.14) since it follows from an elementary combinatorial calculation, and after our choice of parameters the order of magnitude is much smaller than that of the heftiest factor $2^{\frac{4 k \log 3 N}{\delta \log R}}$. Now for $u>0$, we have

$$
\begin{aligned}
\sum_{r=0}^{k+2}(k+r) ! D(k, r) u^{k+r} & \leq u^{k}(k+1)^{2}(k+2)^{2} \sum_{r=0}^{k+2} \frac{k !^{2}(k+r) !^{2}}{r !^{2}(k+2-r) !} u^{r} \\
& =u^{k}(k+2) ! \sum_{r=0}^{k+2} \frac{(k+r) !^{2}}{r !}\left(\begin{array}{c}
k+2 \\
r
\end{array}\right) u^{r} \\
& \leq(2 k+2) !^{2} u^{k}(1+u)^{k+2},
\end{aligned}
$$

so that

$$
I \lesssim N(\log R)^{4(k+\ell)}(\log N)^{2} \frac{(2 k+2) !^{2}}{(k+\ell) !^{4}} 2^{\frac{4 k \log 3 N}{\delta \log R}}\left(\frac{h}{\delta \log R}\right)^{k}\left(1+\frac{h}{\delta \log R}\right)^{k+2}
$$


Using (3.16) and (3.4) in (3.7), we obtain

$$
\begin{aligned}
& \tilde{S}_{R} \lesssim\left(h \sum_{\substack{N<p_{j} \leq 2 N \\
p_{j+1}-p_{j} \leq h}} 1+O\left(N e^{-c \sqrt{\log N}}\right)\right)^{\frac{1}{2}} \\
& \quad \times \frac{(\log R)^{(k+2 \ell)} \log N}{N^{\frac{1}{2}} h^{k}} \frac{(2 k+2) !}{(k+\ell) !^{2}} 2^{\frac{2 k \log 3 N}{\delta \log R}}\left(\frac{h}{\delta \log R}\right)^{\frac{k}{2}}\left(1+\frac{h}{\delta \log R}\right)^{\frac{k+2}{2}} .
\end{aligned}
$$

Now we calculate $\tilde{S}_{R}$ using Propositions 1 and 2. From Proposition 1 and (1.16) we see that

$$
\begin{aligned}
\sum_{N<n \leq 2 N} \log 3 N & \sum_{\mathcal{H}}^{*} \Lambda_{R}(n ; \mathcal{H}, \ell)^{2} \\
& \sim\left(1+O\left(k^{3} \delta^{2}\right)\right)\left(\begin{array}{c}
2 \ell \\
\ell
\end{array}\right) \frac{h^{k}}{(k+2 \ell) !} N(\log R)^{k+2 \ell} \log N .
\end{aligned}
$$

Similarly, Proposition 2 and (1.16) imply

$$
\begin{gathered}
\sum_{\substack{\mathcal{H} \subset[1, h],|\mathcal{H}|=k \\
\mathcal{H}: \text { admissible }}} \sum_{h_{i} \in \mathcal{H}} \sum_{\substack{N<n \leq 2 N \\
\left(P \mathcal{H}(n), \mathcal{P}\left(R^{\delta}\right)\right)=1}} \theta\left(n+h_{i}\right) \Lambda_{R}(n ; \mathcal{H}, \ell)^{2} \\
\sim\left(1+O\left(k^{3} \delta^{2}\right)\right)\left(\begin{array}{c}
2 \ell+2 \\
\ell+1
\end{array}\right) \frac{k h^{k}}{(k+2 \ell+1) !} N(\log R)^{k+2 \ell+1},
\end{gathered}
$$

and

$$
\begin{gathered}
\sum_{\substack{\mathcal{H} \subset[1, h],|\mathcal{H}|=k \\
\mathcal{H}: \text { admissible }}} \sum_{\substack{1 \leq h_{0} \leq h \\
h_{0} \notin \mathcal{\mathcal { H }}}} \sum_{\substack{N<n \leq 2 N \\
\left(P_{\mathcal{H}}(n), \mathcal{P}\left(R^{\delta}\right)\right)=1}} \theta\left(n+h_{0}\right) \Lambda_{R}(n ; \mathcal{H}, \ell)^{2} \\
\gtrsim\left(1+O\left(k^{3} \delta^{2}\right)\right)\left(\begin{array}{c}
2 \ell \\
\ell
\end{array}\right) \frac{h^{k+1}}{(k+2 \ell) !} N(\log R)^{k+2 \ell} .
\end{gathered}
$$

Putting (3.18)-(3.20) together in (3.5) we obtain

$$
\tilde{S}_{R} \gtrsim \frac{\left(\begin{array}{c}
2 \ell \\
\ell
\end{array}\right)}{(k+2 \ell) !}(\log N)(\log R)^{2 \ell}\left\{\frac{k}{k+2 \ell+1} \frac{2(2 \ell+1)}{\ell+1} \frac{\log R}{\log N}+\eta-1+O\left(k^{3} \delta^{2}\right)\right\} .
$$

Now given a small fixed $\eta>0$ if we take

$$
\ell=\left\lfloor\frac{4}{\eta}\right\rfloor, \quad k=2(\ell+1)(2 \ell+1), \quad \delta=\frac{1}{\ell^{4}}, \quad R=N^{\frac{1}{4(1+\delta)}},
$$

then for the factor in brackets in (3.21) we see that

$$
\left\{\frac{k}{k+2 \ell+1} \frac{2(2 \ell+1)}{\ell+1} \frac{\log R}{\log N}+\eta-1+O\left(k^{3} \delta^{2}\right)\right\}>\frac{\eta}{2}
$$

holds for sufficiently small $\eta$, so that $\tilde{S}_{R}>0$.

Noting that (3.2) and (3.22) imply $\frac{h}{\delta \log R}=4 \eta\left(\ell^{4}+1\right)>16 \frac{\ell^{4}+1}{\ell+1} \geq 16$, we will use $1+\frac{h}{\delta \log R}<\frac{2 h}{\delta \log R}$. Then, from (3.17), (3.21) and (3.23), we have

$$
\sum_{\substack{N<p_{j} \leq 2 N \\
p_{j+1}-p_{j} \leq h}} 1 \gtrsim \frac{N}{\log N} \frac{1}{2^{\frac{4 k \log 3 N}{\delta \log R}}} \frac{\left(\begin{array}{c}
2 \ell \\
\ell
\end{array}\right)^{2}(k+\ell) !^{4} \delta^{2 k+2}}{\eta(k+2 \ell) !^{2}(2 k+2) !^{2} 2^{k+10}} .
$$


With the values specified in (3.22), the dominating factor in the coefficient on the right-hand side of (3.24) is

$$
2^{-\frac{4 k \log 3 N}{\delta \log R}}>e^{-65\left(\frac{4}{\eta}\right)^{6} \log 2} .
$$

The other factors in (3.24) give rise to exponents which are $O\left(\frac{1}{\eta^{2}} \log \frac{1}{\eta}\right)$. Thus we obtain

Theorem 2 For sufficiently small but fixed $\eta>0$,

$$
\sum_{\substack{N<p_{j} \leq 2 N \\ p_{j+1}-p_{j} \leq \eta \log N}} 1 \gtrsim e^{-c_{3} \eta^{-6}} \frac{N}{\log N}, \quad(N \rightarrow \infty),
$$

where we can take $c_{3}=\left\lceil 65 \cdot 4^{6} \cdot \log 2\right\rceil=184544$.

(This is not the strongest estimate the present method yields. Taking $\delta=\frac{1}{\ell^{3+c_{4}}}$ with any fixed $c_{4}>\frac{1}{2}$, leads to an estimate of the type (3.26) with $\eta^{-\left(5+c_{4}\right)}$ instead of $\left.\eta^{-6}\right)$.

Note that keeping $Q(N, h)$ all the way down to (3.24), by first keeping it in (3.17) instead of writing the right-hand side of (3.17) via (3.4), yields $Q(N, h) \gg N$, meaning that the proportion of natural numbers $n \in[N, 2 N]$ for which one can find at least two primes within a distance of $h$ from $n$ is positive.

\section{Sparsity of Very SMall gaps BetweEn PRIMES}

The following result expresses that very small gaps between consecutive primes occur rarely, in the sense that such gaps do not constitute a positive proportion of all gaps between consecutive primes.

Theorem 3 For any $h>2$, as $x \rightarrow \infty$, we have

$$
\#\left\{p_{n} \leq x ; p_{n+1}-p_{n} \leq h\right\} \ll \min \left(\frac{h}{\log x}, 1\right) \pi(x) .
$$

In particular, if $h=o(\log x)$, then

$$
\#\left\{p_{n} \leq x ; p_{n+1}-p_{n} \leq h\right\}=o(\pi(x)) .
$$

We remark that for $h=\eta \log x, 0<\eta<1$, the upper estimate for the density of small gaps given by (4.1) corresponds to the conjectured density $1-e^{-\eta}$ from (1.3) apart from the constant implied by the $\ll$ symbol; i.e. the simple upper estimate argument in the proof given below is optimal except for this constant.

Proof: Given two prime numbers $p, p^{\prime}$ satisfying $0<p^{\prime}-p \leq h$, let us write $u+h_{1}=p, u+h_{2}=p^{\prime}$. There are $h$ ordered pairs $\left(u, h_{1}\right)$ with $1 \leq h_{1} \leq h$ such that $u+h_{1}=p$, and for any ordered pair $\left(u, h_{1}\right)$ the value of $h_{2}$ with $h_{1}<h_{2} \leq 2 h$ is fixed. Hence we see that

$$
\begin{aligned}
h \sum_{\substack{N<p, p^{\prime} \leq 2 N \\
0<p^{\prime}-p \leq h}} 1<\sum_{\substack{1 \leq h_{1}, h_{2} \leq 2 h \\
h_{1} \neq h_{2}}} \sum_{\substack{\frac{N}{2}<u<3 N \\
u+h_{1}, u+h_{2}: \text { prime }}} 1 \\
\ll \sum_{\substack{1<h_{1}, h_{2} \leq 2 h \\
h_{1} \neq h_{2}}} \mathfrak{S}\left(\left\{h_{1}, h_{2}\right\}\right) \frac{N}{\log ^{2} N}
\end{aligned}
$$




$$
\ll \frac{h^{2} N}{\log ^{2} N},
$$

where we have used the well-known (see Theorem 5.7 of 9 or Theorem 4 in $\S 2.3 .3$ of [8]) sieve bound for prime tuples

$$
\sum_{N<n \leq 2 N} \theta\left(n+h_{1}\right) \cdots \theta\left(n+h_{k}\right) \lesssim 2^{k} k ! \mathfrak{S}(\mathcal{H}) N
$$

with $k=2$, and Gallagher's result (1.16). Thus we have obtained

$$
\sum_{\substack{N<p, p^{\prime} \leq 2 N \\ 0<p^{\prime}-p \leq h}} 1 \ll \frac{h N}{\log ^{2} N} \ll \frac{h}{\log N} \pi(N) .
$$

We also note that (4.4) used with $k=3$ shows that of the $p, p^{\prime}$ in (4.5), the number of those which are not consecutive is $\ll\left(\frac{h}{\log N}\right)^{2} \pi(N)$.

\section{Conditional Results}

For the circumstance specified by (3.2) we shall now consider the consequence of assuming that the level of distribution of primes $\vartheta$ is greater than $\frac{1}{2}$. The conditions of Propositions 1 and 2 allow us to take

$$
R=N^{\frac{\vartheta-\epsilon}{2(1+\delta)}}
$$

with $\epsilon$ and $\delta$ arbitrarily small fixed positive numbers. We let

$$
\ell=\left\lfloor\frac{\sqrt{k}}{2}\right\rfloor .
$$

For a given $\vartheta>\frac{1}{2}$, we determine $k=k(\vartheta)$ sufficiently large and $\epsilon$ and $\delta$ small enough so as to ensure that the quantity $\frac{k}{k+2 \ell+1} \frac{2(2 \ell+1)}{\ell+1} \frac{\log R}{\log N}-1$ occurring in (3.21) is positive. Now $k$ is not necessarily large enough to satisfy (2.8) and the corresponding inequality when there is the twisting with primes, so instead of the error term $O\left(k^{3} \delta^{2}\right)$ in Propositions 1 and 2, and in (3.21) we will have the cruder $O_{k}(\delta)$ (or else we can re-do the calculation as of (2.4) up until (2.8) without having error terms in what will correspond to (2.5) and (2.6), but this won't be necessary for our purpose). By choosing a smaller $\delta$ if necessary, we will have the factor in brackets in (3.21) (with 0 in place of $\eta$ ) greater than a positive quantity which ultimately depends only on $\vartheta$. Hence, comparing (3.17) and (3.21) we immediately obtain

Theorem 4 Assume that the primes satisfy a level of distribution $\vartheta>\frac{1}{2}$. Let $\eta$ be a fixed positive small number. Then there exists an integer $k(\vartheta)$ and a constant $c_{5}(\vartheta)$ such that

$$
\sum_{\substack{N<p_{j} \leq 2 N \\ p_{j+1}-p_{j} \leq \eta \log N}} 1 \gtrsim c_{5}(\vartheta) \eta^{k(\vartheta)-1} \frac{N}{\log N}, \quad(N \rightarrow \infty) .
$$

Notice that the unconditional estimate (3.26) in which $\eta$ takes place exponentially, gets improved to estimates involving just powers of $\eta$ when it is assumed that the primes satisfy a level of distribution greater than $\frac{1}{2}$. By comparing the factor in brackets in (3.21) with the corresponding factor in the argument in [3], we see that the smallest possible $k(\vartheta)$ we can assert is either the smallest $r=r(\vartheta)$ 
such that every admissible $r$-tuple is guaranteed by the proof of Theorem 1 of 3 ] to contain at least two primes infinitely often or it is $r+1$ (depending on the value of $\vartheta$ ). The greater the level of distribution, the smaller power of $\eta$ will be needed in (5.3). A table of values of $r(\vartheta)$ was provided between (3.4) and (3.5) of [3] (to avoid confusion we have called the $k$ in that table as $r$ here). Thus, if $\vartheta>\frac{20}{21}$, then we can take $k=7, \ell=1$, so that the $\eta$-dependent factor in right-hand side of (5.3) is $\eta^{6}$. However, we recall that assuming $\vartheta \geq 0.971$ and by considering a linear combination of the $\Lambda_{R}(n ; \mathcal{H}, \ell)$ with $k=6$ and $\ell=0,1$, the argument for proving Theorem 1 of [3] still works, so that under this assumption we can get a lower bound in (5.3) which has $\eta^{5}$. We also see from (1.3) that the true order of magnitude of the $\eta$-dependent factor in right-hand side of (5.3) is believed to be $\eta$. Thus for this argument to lead to the true order of magnitude we need to be able to work with admissible pairs (2-tuples). But this seems to require improving the results of [3] to the extent of proving the twin prime hypothesis under the Elliott-Halberstam conjecture.

When $\vartheta$ is slightly greater than $\frac{1}{2}$, from the condition in Proposition 2 , we write

$$
R=N^{\frac{\frac{1}{2}+\xi}{2(1+\delta)}}
$$

where we assume that $\xi>0$ is small. We take

$$
k=2(\ell+1)(2 \ell+1), \quad \delta=\frac{1}{\ell^{4}},
$$

so that

$$
\left\{\frac{k}{k+2 \ell+1} \frac{2(2 \ell+1)}{\ell+1} \frac{\log R}{\log N}+\eta-1+O\left(k^{3} \delta^{2}\right)\right\}=\eta+2 \xi-\frac{1}{\ell}-\frac{2 \xi}{\ell}+O\left(\frac{1}{\ell^{2}}\right)
$$

For a given $\xi$, we determine $\ell$ by

$$
\ell=\left\lceil\frac{1}{\xi}\right\rceil
$$

and then the quantity in (5.6) is $>\eta+\frac{\xi}{2}$ if $\xi$ is sufficiently small. Hence from (3.21) we now have

$$
\tilde{S}_{R}>\frac{\left(\begin{array}{c}
2 \ell \\
\ell
\end{array}\right)}{(k+2 \ell) !}(\log N)(\log R)^{2 \ell}\left(\eta+\frac{\xi}{2}\right)
$$

As before, we derive an upper-bound for $\tilde{S}_{R}$ starting from (3.7), together with (3.8), (3.13) and (3.15). In our case $u=\frac{h}{\delta \log R}$, and upon using the relations in (3.2), (5.4), (5.5) and (5.7), we have

$$
u=\frac{4\left(1+\left\lceil\frac{1}{\xi}\right\rceil^{4}\right)}{1+2 \xi} \eta
$$

This is a small quantity if for a given small $\xi$ we take $\eta$ small enough, say $\eta \leq \frac{\xi^{4}}{5}$, so that we can say $(1+u)^{k+2}<2^{k+2}$. Using this in (3.15) and (3.13) gives

$$
I<N(\log R)^{4(k+\ell)}(\log N)^{2}\left(\frac{h}{\delta \log R}\right)^{k} \frac{2^{\frac{4 k \log 3 N}{\delta \log R}+k+2}(2 k+2) !^{2}}{(k+\ell) !^{4}} .
$$


Plugging this in (3.7), and using that together with (3.4) and (5.8) we obtain (5.11)

$$
\sum_{\substack{N<p_{j} \leq 2 N \\
p_{j}+1 \\
p_{j} \leq h}} 1 \gtrsim \frac{N}{\log N} \eta^{k-1}\left(\frac{\xi}{3}\right)^{2}\left(\frac{4 \delta(1+\delta)}{1+2 \xi}\right)^{k} \frac{\left(\begin{array}{c}
2 \ell \\
\ell
\end{array}\right)^{2}(k+\ell) !^{4} k !^{2}}{(k+2 \ell) !^{2}(2 k+2) !^{2} 2^{\frac{4 k \log 3 N}{\delta \log R}+k+2}} .
$$

By the relations (5.4), (5.5) and (5.7), all of the factors after $\eta^{k-1}$ can be expressed in terms of $\xi$. What interests us most is the power of $\eta$, so we re-express (5.11) as

$$
\sum_{\substack{N<p_{j} \leq 2 N \\ p_{j+1}-p_{j} \leq \eta \log N}} 1 \gtrsim c_{6}(\xi) \eta^{4 \xi^{-2}+14 \xi^{-1}+11} \frac{N}{\log N}, \quad(N \rightarrow \infty)
$$

valid when the level of distribution of primes is assumed to allow us to take $R$ as in (5.4) which can be re-written as

$$
R=N^{\frac{1+2 \xi}{4\left(1+\left\lceil\frac{1}{\xi}\right\rceil^{-4}\right)}}
$$

for fixed $\eta \in\left(0, \frac{\xi^{4}}{5}\right]$. Here $\xi$ has to be sufficiently small, which ensures that $\ell$ and $k$ are sufficiently large so as to permit the inequality (5.8).

In $\S 3$ of [3] it was shown that under the Elliott-Halberstam conjecture we have

$$
\liminf _{n \rightarrow \infty} \frac{p_{n+2}-p_{n}}{\log p_{n}}=0 .
$$

Our method also shows that such gaps occur in positive proportion. To see this we consider

$$
\tilde{S}_{R, 2}:=\frac{1}{N(h \log R)^{k}} \sum_{N<n \leq 2 N}(\Theta(n, h)-2 \log 3 N)\left(\sum_{\mathcal{H}}^{*} \Lambda_{R}^{2}(n ; \mathcal{H}, \ell)\right) .
$$

As was done in [3] along with the modification provided by (2.8), we find

$$
\tilde{S}_{R, 2} \gtrsim \frac{\left(\begin{array}{c}
2 \ell \\
\ell
\end{array}\right)}{(k+2 \ell) !}(\log N)(\log R)^{2 \ell}\left\{\frac{k}{k+2 \ell+1} \frac{2(2 \ell+1)}{\ell+1} \frac{\log R}{\log N}+\eta-2-k^{3} \delta^{2}\right\} .
$$

Here we are assuming that $\vartheta=1$, and so we can take $R=N^{\frac{1}{2(1+\delta)}}$. In the proof of (5.14), $k$ is taken to be sufficiently large, $\ell=\left\lfloor\frac{\sqrt{k}}{2}\right\rfloor$. If $\delta$ is taken to be accordingly small, say $\delta=\frac{1}{k^{2}}$, then the quantity in brackets in (5.16) is

$$
\begin{aligned}
& >2\left(1-\frac{2 \ell+1}{k}-\frac{1}{2 \ell}\right)(1-\delta)+\eta-2-k^{2} \delta^{3} \\
& >\eta-\frac{2(2 \ell+1)}{k}-\frac{1}{\ell}-2 \delta-k^{2} \delta^{3} \\
& >\eta-\frac{2(\sqrt{k}+1)}{k}-\frac{2}{\sqrt{k}-2}-\frac{2}{k^{2}}-\frac{1}{k} \\
& >\eta-\frac{5}{\sqrt{k}}-\frac{3}{k}-\frac{2}{k^{2}} \quad(\text { for } k>36) \\
& >\eta-\frac{6}{\sqrt{k}} \\
& >\frac{\eta}{2} \quad\left(\text { for } k>\frac{144}{\eta^{2}}\right) .
\end{aligned}
$$


The rest of the argument is almost identical to what was done as of (3.7), the only changes are that we now have the summation condition $\Theta(n, h) \geq \frac{5}{2} \log N$, and $\frac{h}{\delta \log R}=2 \eta\left(1+\frac{1}{\delta}\right)$ being not small we should use some bound like $(1+u)^{k+2} \leq$ $(2 u)^{k+2}$ (cf. between (5.9) and (5.10)). The following is the result of this calculation.

Theorem 5 Assuming the Elliott-Halberstam conjecture we have

$$
\sum_{\substack{N<p_{j} \leq 2 N \\ p_{j+2}-p_{j} \leq \eta \log N}} 1 \gtrsim e^{c_{7} \eta^{-2} \log \eta} \frac{N}{\log N}, \quad(N \rightarrow \infty)
$$

$\left(c_{7}=5\right.$ gives a valid result if $\eta$ is small enough).

\section{REFERENCES}

[1] E. Bombieri and H. Davenport, Small differences between prime numbers, Proc. Roy. Soc. Ser. A 293 (1966), 1-18.

[2] P. X. Gallagher, On the distribution of primes in short intervals, Mathematika 23 (1976), $4-9$.

[3] D. A. Goldston, J. Pintz and C. Y. Yıldırım, Primes in tuples I, Ann. of Math. 170 (2009), 819-862.

[4] D. A. Goldston, J. Pintz and C. Y. Yıldırım, Primes in tuples II, Acta Math. 204 (2010), $1-47$.

[5] D. A. Goldston, J. Pintz and C. Y. Yıldırım, Positive proportion of small gaps between consecutive primes, Publ. Math. Debrecen (to appear).

[6] D. A. Goldston and C. Y. Yıldırım, Higher correlations of divisor sums related to primes I: Triple correlations, Integers 3 (2003), A5, 66pp. (electronic).

[7] D. A. Goldston and C. Y. Yıldırım, Higher correlations of divisor sums related to primes III: Small gaps between primes, Proc. London Math. Soc. (3) 95 (2007), no. 3, 653-686.

[8] G. Greaves, Sieves in number theory, Springer, Berlin, 2001.

[9] H. Halberstam and H.-E. Richert, Sieve methods, Academic Press, New York, 1974.

[10] M. N. Huxley, Small differences between consecutive primes II, Mathematika 24 (1977), 142152.

[11] H. Maier, Small differences between prime numbers, Michigan Math. J. 35 (1988), 323-344.

[12] J. Pintz, Are there arbitrarily long arithmetic progressions of twin primes?, in An irregular mind, Szemerédi is 70, (Editors: I. Barany and J. Solymosi), 525-559, Bolyai Soc. Math. Studies 21, Springer, 2010.

[13] J. B. Rosser and L. Schoenfeld, Approximate formulas for some functions of prime numbers, Illinois J. Math. 6 (1962), 64-94.

[14] K. Soundararajan, Small gaps between prime numbers: The work of Goldston-Pintz-Yıldırım, Bull. Amer. Math. Soc. (N.S.) 44 (2007), 1-18.

[15] K. Soundararajan, The distribution of prime numbers. Equidistribution in number theory, an introduction, 59-83, NATO Sci. Ser. II Math. Phys. Chem., 237, Springer, Dordrecht, 2007. 
D. A. Goldston (goldston@math.sjsu.edu)

Department of Mathematics

San Jose State University

San Jose, CA 95192

USA

J. Pintz (pintz@renyi.hu)

Rényi Mathematical Institute of the Hungarian Academy of Sciences

H-1364 Budapest

P.O.B. 127

Hungary

C. Y. Yıldırım (yalciny@boun.edu.tr)

Department of Mathematics

Bog̃aziçi University

Bebek, Istanbul 34342

Turkey 